\title{
Pollination of Dionaea muscipula, the Venus Flytrap
}

\author{
Stephen E. Williams • Emeritus Professor • Lebanon Valley College • Annville • Pennsylvania \\ -USA・williams@lvc.edu \\ BILL SCHOLL • Liberty County・Florida・USA・scholljunk@gmail.com
}

Abstract: Dionaea flowers are pollinated by insects, primarily bees, in both their native habitat in the Carolinas and in the population growing from seed scattered many years ago in the Florida panhandle, the location of this study. The green sweat bee (Augochlorella sp. is a major pollinator in both habitats, while various flower visiting beetles play a prominent role in the Carolinas and bumblebees (Bombus sp.) play a major role in the Florida population.

Flowers open in late morning and pollen is on anthers arrayed directly above the center of the flower by 11:30 EST. Twenty-four hours later the stamens have bent so that the anthers are at the periphery of the flower and the stigma has expanded, presenting a papillate surface receptive to pollen. Insects visit the flower between 11:30 EST and 18:00 EST.

Rewards for pollinators are pollen and nectar from glands at the base of the petals. Possible attractants include the bright white radially symmetrical corolla with radiating grey veins, and on the second day, the radially displayed stamens that may act as a "nectar guide". No markings on the petals are visible in the ultraviolet light but the pollen-laden anthers are UV-fluorescent and would stand out as a ring of dots to any UV-perceptive insect.

\section{Introduction}

There have been two notable contributions to the pollination ecology of Dionaea muscipula, both made in its native habitat in the Carolinas. The first, by Roberts and Oosting (1958), established the dates of flowering, its induction by photoperiod, and the development of flowers, as well as proposing possible pollinators based on observed floral visitors. The second by Youngsteadt et al. (2018) is a thorough quantitative examination of the visitors and pollinators of Dionaea finding the most common pollinators to be two species of small solitary bees and several species of beetles that specialize in pollination. The primary objective of Youngsteadt et al. (2018) was to determine if Dionaea pollinators would be preyed upon by the traps. They were not.

The inflorescence of Dionaea is an umbelliform cyme with 2 to 14 flowers on a long scape that arises from a small perennial rhizome (Smith 1929; Radford et al. 2010). Roberts and Oosting (1958) observed that the flowers of Dionaea begin opening at the terminal flower of inflorescences that bear an average of six flowers on a scape about 20-30 cm long, well away from the traps. They state that at the time a flower opens from its bud its anthers are shedding pollen. Approximately 24 hours later the stigma is receptive. They also performed experiments that indicated the flowers do not self-pollinate.

Roberts and Oosting (1958) suggested visitors that they observed on flowers as possible pollinators. These included "various beetles, small flies and possibly spiders".

The much more thorough investigation of the pollinators of Dionaea by Youngsteadt et al. (2018) revealed that its major pollinators are the sweat bee Augochlorella gratiosa (Halictidae) which had the highest relative pollen abundance, and the longhorned beetle Typocerus sinuatus (Cerambycidae) and the checkered beetle Trichodes apivorus (Cleridae) which carried the largest pollen load. 
Their study carefully documents the pollinator's identity and measures effectiveness but does not look at timing of pollination, the role in pollination of the changes in flower structure that result in protandry (Roberts \& Oosting 1958), or other characteristics of the flower that are involved in pollination. Their efforts were concentrated on the insect pollinators.

The present report results from observations on Dionaea flowers made in the course of a quantitative study of captures by the traps. The observations include the timing of floral development and pollination of Dionaea muscipula flowers and the changes in flowers during anthesis.

Dionaea was introduced into the area around Hosford, Liberty County, Florida in 1973 or 1974 by a carnivorous plant collector and have thrived there in the Florida coastal plain savannas since that time. A different population of Dionaea was introduced independently 20 miles south of Hosford about 2003 in a coastal savanna area similar to the plant's native habitat in the Carolinas. These plants, growing on private land within the Apalachicola National Forest since 2003, were used in this study. ${ }^{1}$

This study describes in detail the timing of the protandrous development of the Dionaea flowers and of pollinator visits. It also allows a comparison of pollination in the coastal savannas of the Florida Panhandle with those in the Carolinas.

\section{Methods}

Plants in a stand of Dionaea muscipula established near Sumatra, Florida on private land within the Apalachicola National Forest were observed while in flower between 4 May 2020 and 21 May 2020.

Video of a $24.5 \times 15 \mathrm{~cm}$ area was recorded using a time-lapse mechanism to record 1-min videos every five minutes using the $1920 \times 1080$ video setting of a Bushnell NatureView CAM with a 460 $\mathrm{mm}$ close up lens. The camera was mounted horizontally, looking directly down on the flowers, using an RC Williams Co. TopView Quick-Mount. The camera mount held the camera firmly in place so that it could be set in the exact same location after changing the SD card. Thus, one of every five minutes was sampled during a 43-hour period.

The videos were then observed continuously with a VLC Media Player, noting any activity by visitors.

Flower development was observed in the time-lapse videos and the changes in form of the flowers over three days were noted. More detailed images of the flower development were recorded using a Celestron Handheld Digital Microscope Pro 5mp.

Ultraviolet photography of flowers from plants cultivated by Siegfried Hartmeyer was done using a Raytech Versalume UV-lamp with two glass filters for $254 \mathrm{~nm}$ and $366 \mathrm{~nm}$. The fluorescence resulted from a $366 \mathrm{~nm}$ excitation and was photographed with an Olympus SH-2 camera.

\section{Results and discussion}

Anthesis began in the morning between 10:00 (EST) and noon.

First day flowers developed from buds that had swollen to about twice their previous size overnight (Figs. 1a, 1b) and then open in less than an hour during late morning (Figs. 1b, 1c). The anthers

\footnotetext{
${ }^{1}$ The neighboring Florida population in this study originated from seed, descended from plants from the Carolinas, broadcast over the savannah habitat in the Florida panhandle 15 to 17 years ago. The seeds descend from Dionaea plants grown in Virginia from seed originally harvested from the Carolinas about 40 to 50 years ago. All plants were from broadcast seed except for the transplanted plants mentioned in the text (Observed by Bill Scholl).
} 

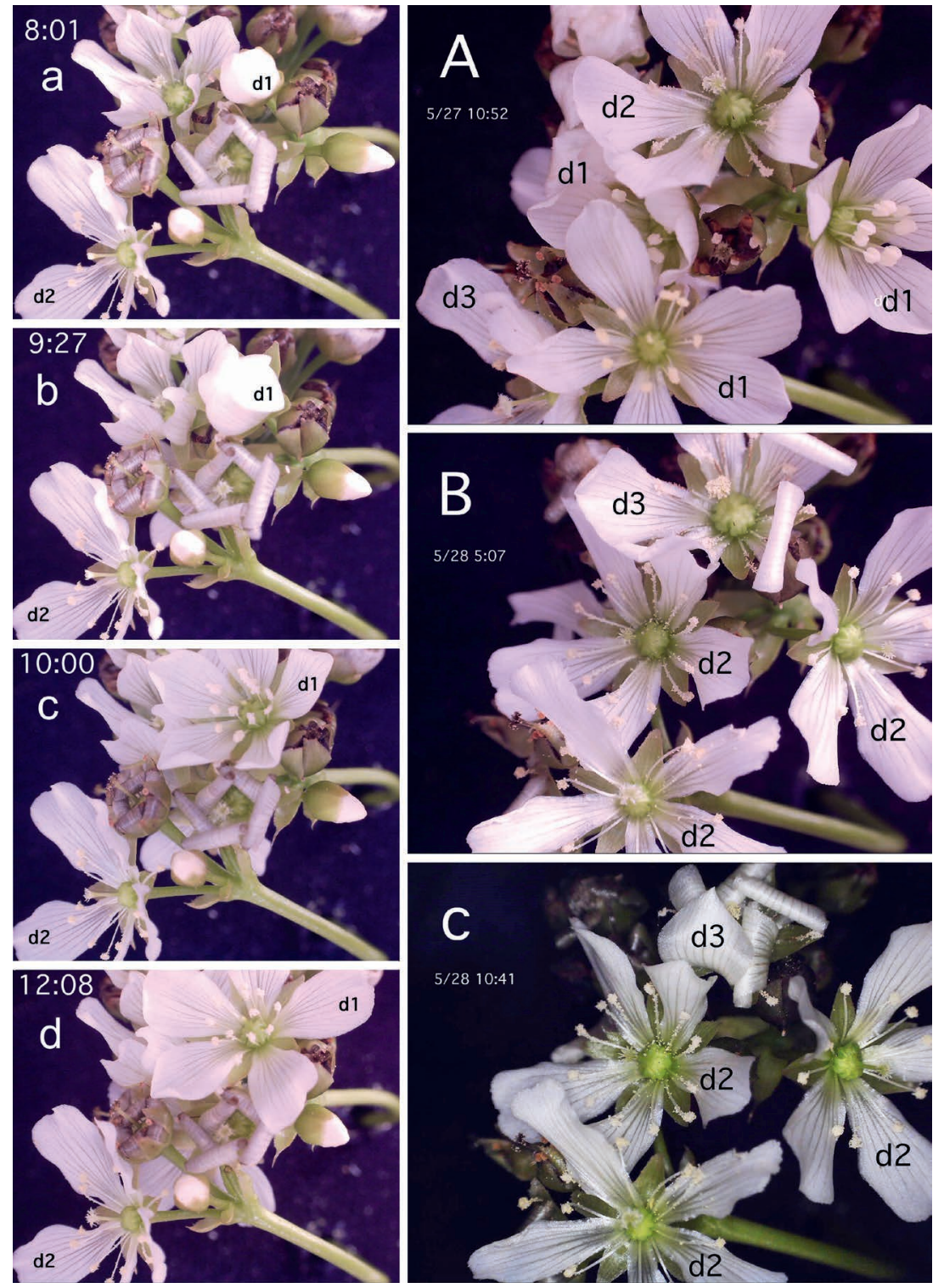

Figure 1: (left): Flower development: Time of photograph is given in the upper left corner in 24-hr time EST. Flowers opening from buds on day illustrated = $\mathrm{d} 1$. Flowers that opened previous day $=\mathrm{d} 2$. Note anther development on $\mathrm{d} 1$ flowers and stigma development on d2 flowers.

Figure 2: (right): Protandrous flower development: The date and time (24-hr time EST) is in the upper left-hand corner. This shows the same inflorescence on two different days, illustrating flowers as day-one flowers in $\mathrm{A}$ and day-two flowers in B and $\mathrm{C}$. Note the differences between day-one flowers (d1) and daytwo (d2) flowers in B and C. The day-2 flower in A is a day-3 flower in B and C. 


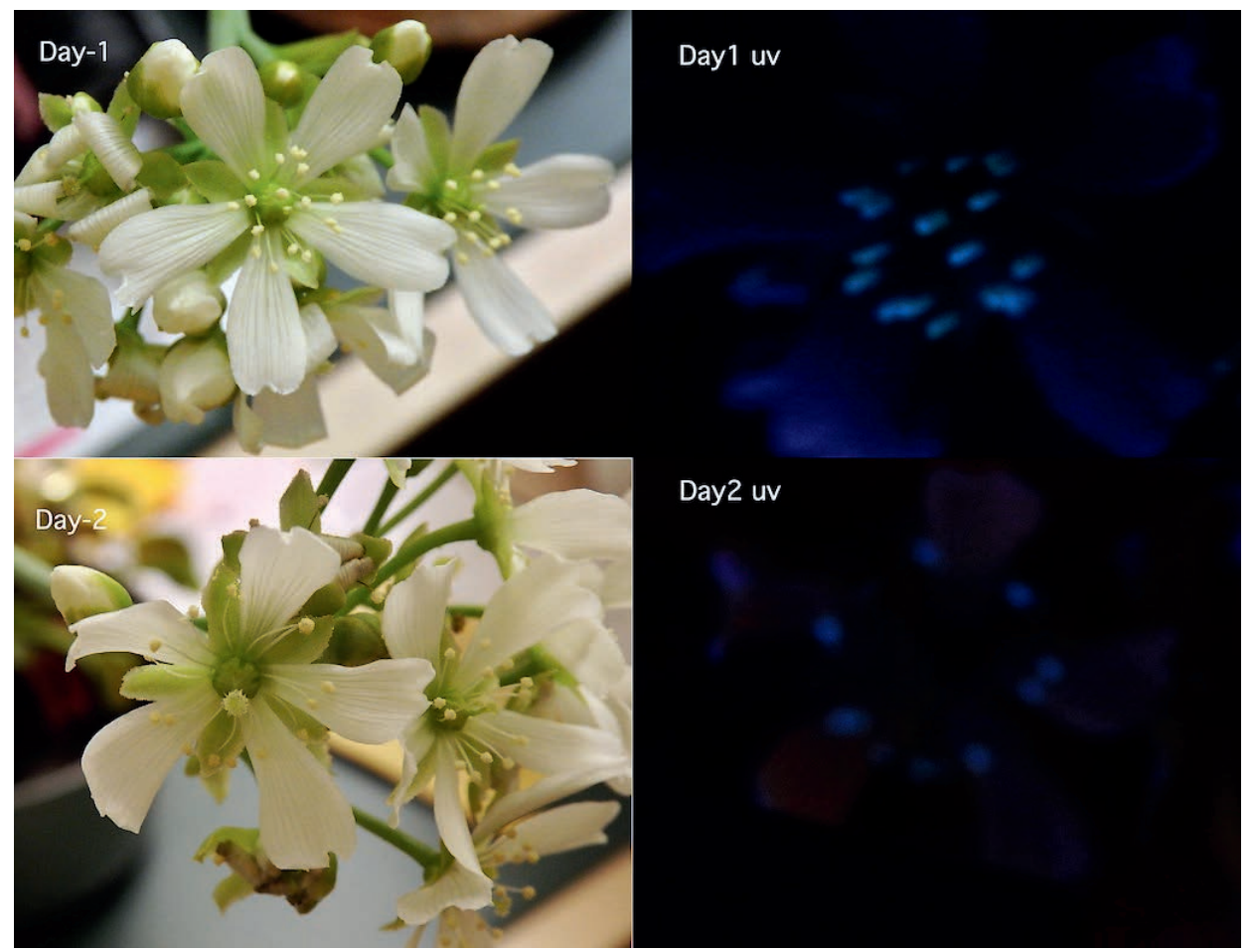

Figure 3: In the laboratory the petals of both day-one (top) and day-two (bottom) flowers only weakly reflect ultraviolet and show no patterns visible in these wavelengths. By contrast the anthers, probably due to fluorescence by the pollen, appear as a series of dots in the center of the day-one flower (top). The anthers of the day-two flowers (bottom) show as a weaker ring of dots. This ring is absent from flowers out of doors where the pollen is stripped from the anthers by insects.

dehisce about 45 min later (Fig. 1d). By 12:00 EST the flower is fully open and the five radially arranged petals are tilted slightly toward the center of the flower; the stamens are erect so that the anthers are arrayed over the center of the flower (Figs. 1d, 2A); and the style is short with the immature stigma appearing as a small green knob (Figs. 1d, 2A, 3 day 1). In the evening (about 16:00 EST) the stamens bend backward toward the petals and the petals move slightly upward. This results in the stamens being arrayed radially with two over each petal (Fig. 2B).

Second day flowers in the morning appeared unchanged from the previous evening except for the elongation of the style which bears a small, undeveloped stigma. Between 10:00-12:00 the stigma rapidly expands to about three times its previous diameter and presents a highly papillate surface (Fig. 1c and 1d).

Third day flowers appear similar to the previous evening except that the papillate stigmas are much wider and the petals are flexed further upward with the stamens pressed against them. At the end of the third day the petals and stamens deteriorate and fruit development begins (Figs. 2B, 2C).

Visits by insects to the flowers occurred between 11:30 and 18:00 EST with the exception of the small black wasp that once appeared shortly after 9:30. It was feeding on pollen remaining in a day-two flower. 


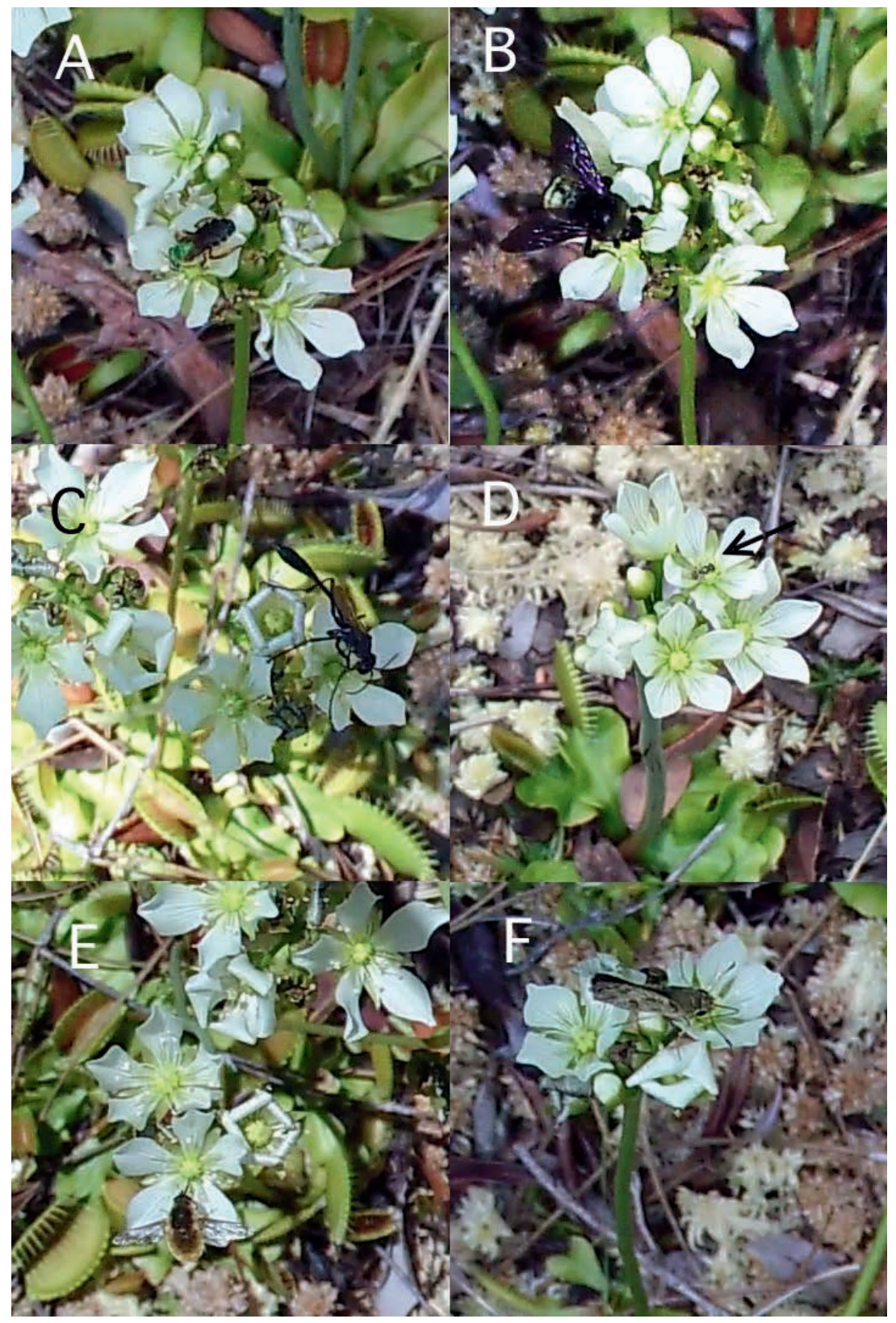

Figure 4: Insect visitors: A. A green sweat bee (Augochlorella sp) working the nectar glands at the base of the petals of a flower while pushing against its stigma. B. A bumblebee (Bombus sp) taking nectar from the same flower. C. A thread-waisted wasp $(\sim 1.4 \mathrm{~cm}$; Sphecidae $)$ at nectar glands. D. A small wasp $(\sim 3 \mathrm{~mm})$ visits the nectar glands of a flower (see arrow). E. A bee fly (Bombylidae) probes a nectar gland at the base of a petal with its proboscis. F. A butterfly (Hesperiidae) rests on the petals of a flower while probing the nectar glands. 
Six species of frequent visitors were observed repeatedly visiting flowers; two kinds of bees, two species of wasps, a species of bee fly, and one type of butterfly (Fig. 4). Several other small insects were occasionally seen resting on petals but did not approach anthers or stigmas.

The two bees were a green sweat bee (Augochlorella sp., Fig. 4A) and a bumblebee (Bombus sp., Fig. 4B). Augochlorella gratiosa was by far the most important pollinator of Dionaea in its native range in the Carolinas, accounting for a larger pollination index than the sum of all other pollinators combined, while Bombus was not reported as a pollinator (Youngsteadt et al. 2018). In the present study, in Florida, both bee species interacted vigorously with both the pollen-presenting day-one flowers and the stigma-receptive day-two flowers and both species of bee actively sought both nectar and pollen.

The two wasps were a thread-waisted wasp $(\sim 1.4 \mathrm{~cm})$ (Sphecidae, Fig. $4 \mathrm{C})$ and a small black wasp ( $\sim 3 \mathrm{~mm}$ ) (Fig. 4D). Both visited day-one and day-two flowers and touched both pollen bearing anthers and receptive stigmas. The thread-waisted wasp sipped nectar and moved deftly around the flowers performing all the actions a pollinator would but it is not clear that its smooth surface would carry pollen. The small wasp walked around the surface, sometimes eating pollen remaining on day-two anthers and sometimes sipping nectar. It touched both the stigma and anthers at times but the timing was not the same as the other visitors and their motions seemed helter-skelter. It is possible that they delivered a few pollen grains to a few receptive stigmas but they are not likely to be a major pollinator. Further investigation is necessary for both of these insects.

The bee fly and butterfly visited both day-one and day-two flowers. The hovering bee flies (Bombylidae, Fig. 4E) reached the nectar glands from the side with their long mouthparts, avoiding the stigmas, but sometimes coming in contact with the anthers. The butterflies (Hesperiidae, Fig. 4F), which sat on petals as they extracted nectar, had mouthparts that slipped past the anthers as well as the stigma. Both of these species are nectar thieves.

All species of insects that visited the flowers during anthesis were capable of both flight and of extracting nectar from the flowers. All six species of visitors visited both day-one, pollen bearing, and day-two receptive stigma bearing flowers. Bees and wasps made contact with both anthers and stigmas but the bee flies and butterflies introduced their long mouthparts from the side, avoiding the stigmas. The butterfly, which sat on several petals, had mouthparts that were long enough to slip past the anthers as well (Figs. 4E, 4F).

The timing of visits correlates perfectly with the availability of pollen and the receptivity of stigmas.

\section{Comparison of pollinators with Youngsteadt et al. (2018)}

The green sweat bee (Augochlorella gratiosa) was the major pollinator of Dionaea growing in its natural habitat studied by Youngsteadt et al. (2018) and a species of Augochlorella is clearly an important pollinator of the introduced Florida plants that we studied. Bumblebees (Bombus spp.) are not mentioned by Youngsteadt et al. (2018) although it is certainly an important pollinator in the Florida plants. The thread-waisted wasp (Sphecidae) is also not mentioned but this may be because it was a visitor that carried no pollen and thus did not make their list of pollinators. The same may be true of all visitors we observed other than the two bees. By contrast we observed none of the beetles that were such an important part of the pollination biology of Dionaea in the Carolinas.

Although the habitats are very similar, several caveats remain. First, the fauna in the Florida panhandle and the Carolinas are almost certainly not exactly the same. Second, our sample size is smaller than in the more extensive study of Youngsteadt et al. (2018). 
The terminal flowers of the inflorescence develop first with flowers on subordinate branches developing sequentially.

The protandrous sequence of flower opening, described previously, has individual flowers with viable pollen the first day and receptive stigmas the second. This explains the self-incompatibility of flowers observed by Roberts and Oosting (1958), provided that the pollen remaining on the anthers is not viable the second day.

Our observations on the inflorescence and flower development agree with the brief, limited, descriptions by C.M. Smith (1929) and Roberts and Oosting (1958) except for Roberts and Oosting's statement that a first day flower "immediately begins shedding pollen" upon opening. There is actually a period of about $45 \mathrm{~min}$ between the opening of a bud and the dehiscence of the anthers.

The result of the developmental sequence of Dionaea flowers is that day-one flowers have anthers bearing free pollen, displayed prominently in the center of the flower in late morning, about 11:30 EST on the first day. Approximately 24 hours later at about 11:30 EST the anthers of the flower, now in day-two form, are away from the center of the flower and the newly enlarged stigma occupies a prominent place in the center. The anthers and stigmas are both optimally positioned for pollination on successive days.

\section{Attractants}

Pollinators drawn to the pollen-laden anthers of the day-one flowers are rewarded with both pollen and nectar. The day-two flowers, usually stripped of pollen, still provide a nectar reward. The flowers are also conspicuous, with attractants common to many flowers such as a radially symmetrical array of petals and stamens, and possibly a scent during the period of anthesis.

Pollen is a reward that is prominently displayed on day one with the dehisced anthers appearing in ultraviolet as a cluster of dots in the center of the flower (Fig. 3). Pollen has usually been stripped by day two but if it remains, the positioning of the anthers to the side would result in a circle of dots on the corolla in ultraviolet (Fig. 3).

Nectar glands at the base of the petals are active attractants on both day-one and day-two. The bees and wasps (Figs. 4A, 4B, 4C, 4D) take nectar from the base of the petals and the nectar glands are clearly probed by the long proboscis of bee flies visiting the flowers (Fig. 4E).

Petal and Stamen display is a feature that changes gradually as the flowers age.

Five bright white petals with about eight vertical gray veins on each petal radiate from the center of the flower. There is only a small amount of ultraviolet fluorescence from the petals (Fig. 3). On the evening of day-one and in day-two flowers when the stamens move toward the petals their filaments add a new set of radiating lines to the flowers' appearance. In the laboratory the pollen adhering to the anthers still reflects a radial pattern but in the field the anthers have typically been stripped of their pollen. On day two and day three the broad white stigma stands out in the center of the flower.

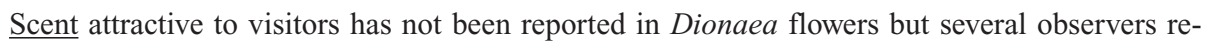
ported smelling an odor during the period of pollination. This needs further investigation.

\section{Seeds}

The population of Dionaea in the present pollination study produces viable seeds. The population originated from 3 or 4 plants transplanted from a neighboring Florida population about fifteen 
years ago. The population has subsequently spread over about $1.5 \mathrm{~m}^{2}$, probably from its own seed, since the plants are varying size and characteristics, as expected from a sexually reproduced population. The plants produce abundant seed every year (Scholl, Pers. obs., 2020).

The pollinators in this study clearly are causing successful pollinations resulting in viable seeds.

Acknowledgments: We would like to thank Dr. Susan Verhoek of Lebanon Valley College for help in framing the study and constructive comments on the research. We thank Dr. Orland Blanchard of the University of Florida Herbarium for valuable suggestions for improving the manuscript and Dr. Allan Wolfe of Lebanon Valley College for aid in identifying the insects. Siegfried Hartmeyer of Weil am Rhine, Germany, provided information on ultraviolet absorption and reflection of the flowers and the photographs in Figure 3.

\section{References}

Radford, A.E., Bell, C.R., and Ahles, H.E. 2010. Manual of the Vascular Flora of the Carolinas.

Univ. of North Carolina Press, Chapel Hill, NC.

Roberts, P.R., and Oosting, H.J. 1958. Responses of Venus fly trap (Dionaea muscipula) to factors involved in its endemism. Ecol. Monographs 28: 193-218.

Smith, C.M. 1929. Development of Dionaea muscipula, I. Flower and seed. Bot. Gaz. 87: 507-530. Miller, J. 2019. The Florida flytrap mystery is solved. Carniv. P1. Newslett. 48(3):135.

Youngsteadt, E., Irwin, R.E., Fowler, A., Bertone, M.A., Giacomini, S.J., Kunz, M., Suiter, D., and Sorenson, C.E. 2018. Venus flytrap rarely traps its pollinators. Am Nat. 2018 Apr;191(4): 539546. doi: 10.1086/696124. Epub 2018 Feb 5. PMID: 29570403.

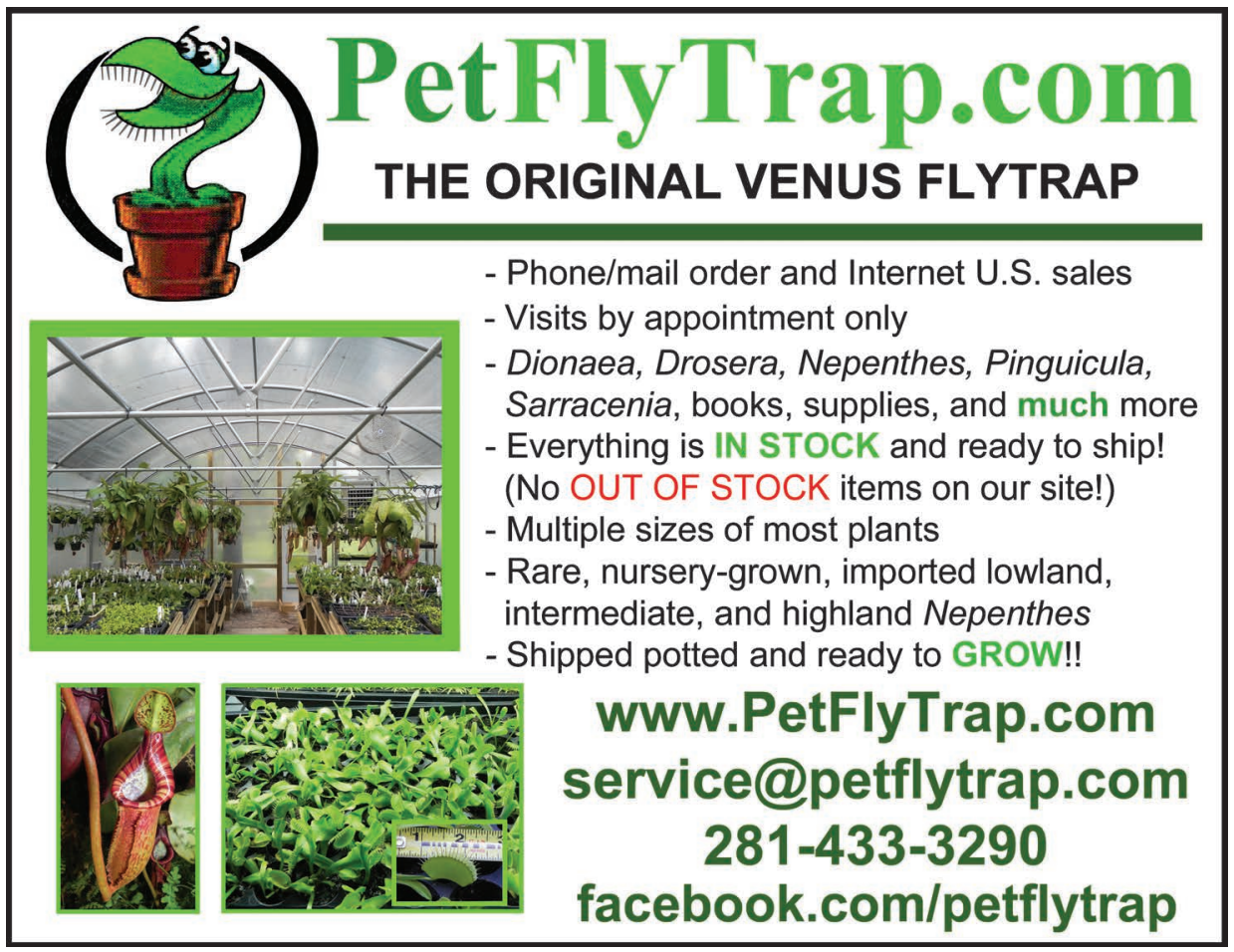

